American Journal of Nursing Science
2020; $9(2): 74-78$
http://www.sciencepublishinggroup.com/j/ajns
doi: 10.11648 /j.ajns.20200902.17
ISSN: 2328 -5745 (Print); ISSN: $2328-5753$ (Online)

\title{
Nurses' Psychological Feelings About the Application of Gibbs Reflective Cycle of Adverse Events
}

\author{
Yanfei $\mathrm{Li}^{1}$, Weiju Chen ${ }^{2,}$, Cuiqing Liu ${ }^{3}$, Minping Deng ${ }^{4}$ \\ ${ }^{1}$ Department of Spinal and Trauma, The First Affiliated Hospital of Jinan University, Guangzhou, China \\ ${ }^{2}$ School of Nursing, Jinan University, Guangzhou, China \\ ${ }^{3}$ Department of Nursing, The First Affiliated Hospital of Jinan University, Guangzhou, China \\ ${ }^{4}$ Department of Gastrointestinal Surgical, The First Affiliated Hospital of Jinan University, Guangzhou, China
}

Email address:

chenweiju@126.com (Weiju Chen)

${ }^{*}$ Corresponding author

\section{To cite this article:}

Yanfei Li, Weiju Chen, Cuiqing Liu, Minping Deng. Nurses' Psychological Feelings About the Application of Gibbs Reflective Cycle of Adverse Events. American Journal of Nursing Science. Vol. 9, No. 2, 2020, pp. 74-78. doi: 10.11648/j.ajns.20200902.17

Received: March 2, 2020; Accepted: March 18, 2020; Published: April 1, 2020

\begin{abstract}
Subject: To investigate the psychological feelings of nurses about the application of Gibbs Reflective Cycle during nursing adverse events. Methods: 13 cases were interviewed in depth by semi-structured interviews, and the interview data were analysed and summarized. Result: After the occurrence of adverse events, nurses on duty have negative emotions, especially in the event of big adverse events, the psychological feeling of depression is more intense, and even leading to the new nurses on duty doubt about their careers. The nurses think that the Gibbs Reflective Cycle is helpful for the growth, especially for nursing staff with low seniority. However, it is difficult to determine the specific cause when analyzing the cooperation and participation of adverse events. Nurses also worry that a reflective diary could become a bad record for the rest of their careers. Conclusion: Gibbs reflection Cycle's theoretical approach can help nurses develop clinical nursing thinking in the process of dealing with adverse events and deepen nurses' understanding of the causes of adverse events. At the same time, nursing managers should pay attention to nurses' negative psychological state and eliminate their concerns about poor work records. Using Gibbs reflection Cycle to reflect on the causes of adverse events is a way to develop nurses' clinical thinking, which is an effective way to continue medical education and training.
\end{abstract}

Keywords: Gibbs Reflective Cycle, Nursing, Adverse Events, Qualitative Research

\section{Introduction}

Reflection is a useful and valuable tool for professional development in nursing and is used widely as a teaching and learning methodology for nursing students both in the classroom and during nursing practice [1]. Common theories of reflection include Atkins and Murphy Reflective Cycle (1994), The Gibbs Reflective Cycle (1988) and john's Model of Structured Reflection (1996). The Gibbs Reflective Cycle (1988) is also one of the three common reflective theories, which has been widely applied in the education and practice of medical students [2].

The Gibbs Reflective Cycle can apply to any scenario that you feel merits reflection and/or improvement. This cycle is used in the health-care profession as a way for professionals to assess and reflect on any given situation in their job. By reflecting, and learning to do it reflexively, the professional can gain a sense of how a method or his or her actions can be improved upon when a similar situation arises. The classic Gibbs Reflective Cycle consists of 6 parts, namely description, feeling, evaluation, analysis, conclusion and action plan [3].

Most of people's knowledge and understanding are acquired through reflection through experience. Continuous thinking can reflection better identify things and guide the next step of the plan. This is where Gibbs Reflective Cycle is useful. Scholars at home and abroad have applied the Gibbs Reflective Cycle to the education of clinical nursing students 
in order to improve their professional knowledge development and emotional field training [4-5]. Recent research has also focused on analyzing the correlation between an implementation case reflection discussion (CRD) based on the Graham Gibbs Cycle, and nurses' critical thinking skills. The purpose of the study is to Case reflection discussion (CRD) based on the Graham Gibbs Cycle increased nurses' critical thinking skills [6].

However, the theoretical framework of Gibbs Reflective Cycle has not been applied to the analysis process of clinical adverse events and the clinical study of clinical nurses' psychological experience. The qualitative research method that Gibbs Reflective Cycle tool were used to study the influence of adverse events on nurses' clinical nursing thinking and psychological cognition. Qualitative research method can elaborate the real inner experience of nurses in detail, which is helpful to understand the inner feelings of nurses when Gibbs Reflective Cycle is used to analyze adverse events.

\section{Method}

\subsection{Participants}

Using the method of target sampling, the nurses working in the spinal ward of a third-grade a hospital in Guangzhou were interviewed in 2019. Inclusion criteria: Nurses who are engaged in clinical nursing work and have had more than one case of adverse events before and after using the Gibbs Reflective Cycle are willing to participate in this study after training on reporting and handling adverse events in the department.

\subsection{Procedure}

All participants completed a face-to-face, semi-structured, interview. Interviews were analyzed following the procedure of Interpretative-Phenomenological Analysis (IPA) [7]. The interview was conducted after the daily work. All interviews were performed face-to-face by the first author in a quiet location convenient to the survivor. All interviews were audio-recorded with consent. The interview lasted an average of $35 \mathrm{~min}$, ranging from 19 to $50 \mathrm{~min}$. The information saturated sample size is appropriate. Before the interview, the researcher introduces the purpose and significance of the study to the research object, and obtains the consent of the interviewee for the recording question. In order to protect the privacy of patients, all the research content is anonymous and the name is numbered instead.

\subsection{Data Collection}

A semi-structured, interview was developed for this study that included open-ended questions such as "How did you feel when you learned that the patient you were responsible for had an adverse event?" "What do you think of the Gibbs
Reflective Cycle theory?" "What do you think of using the Gibbs Reflective Cycle to write reflective diaries after an adverse event?" "What difficulties do you have in writing?" "How to deal with difficulties?". Matters needing attention, context and non-verbal communication during the interview should be recorded in the memo.

\subsection{Data Aanalysis}

Data collation and analysis should be carried out simultaneously. Use content analysis and data analysis, including text transcription, finding analytical units, coding, categorizing, presenting topics, and validating topics. Using memos to record data requires thinking and summarizing during analysis, Memoranda should be integrated with the data analysis process. Based on the key words recorded in the interview and the expression, tone, eyes, silence time and body movements of the interviewees, the written materials were repeatedly formed to extract statements of great significance. The two researchers coded and classified independently, extracted themes from which, compared and discussed data analysis results. Different opinions reached consensus through discussion and reflection.

\subsection{Ethical Issues}

The research may involve personal privacy, but researchers do the following four things when it comes to ethical issues: a, The purpose and significance of the research are introduced to the interviewees before the interview; $b$, The interview was recorded with the consent of the researcher; c, Subjects have the right to withdraw from the study at will, without being unfairly treated; d, After the interview, the client should be encouraged and encouraged based on the new hope.

\section{Results}

A total of 13 interviewees were included in this study, of none withdrew from this study. The interviewees of the study were aged between 23 and 35, all female, with clinical working time between 1 and 12 years, and working experience less than 5 years accounted for $69 \%$. Their educational background was divided into technical secondary school, junior college and bachelor's degree (table 1). Almost all the interviewees experienced different psychological emergency reactions such as fear, depression, fear, worry and chagrin when they were informed of the occurrence of adverse events, and some even appeared shame and other problems. Against this background of psychological pressure, when nurses use the Gibbs Reflective Cycle to review and analyze adverse events, Nurses with different qualifications showed different feelings and behaviors in different types of adverse events. 
Table 1. Information of the interview.

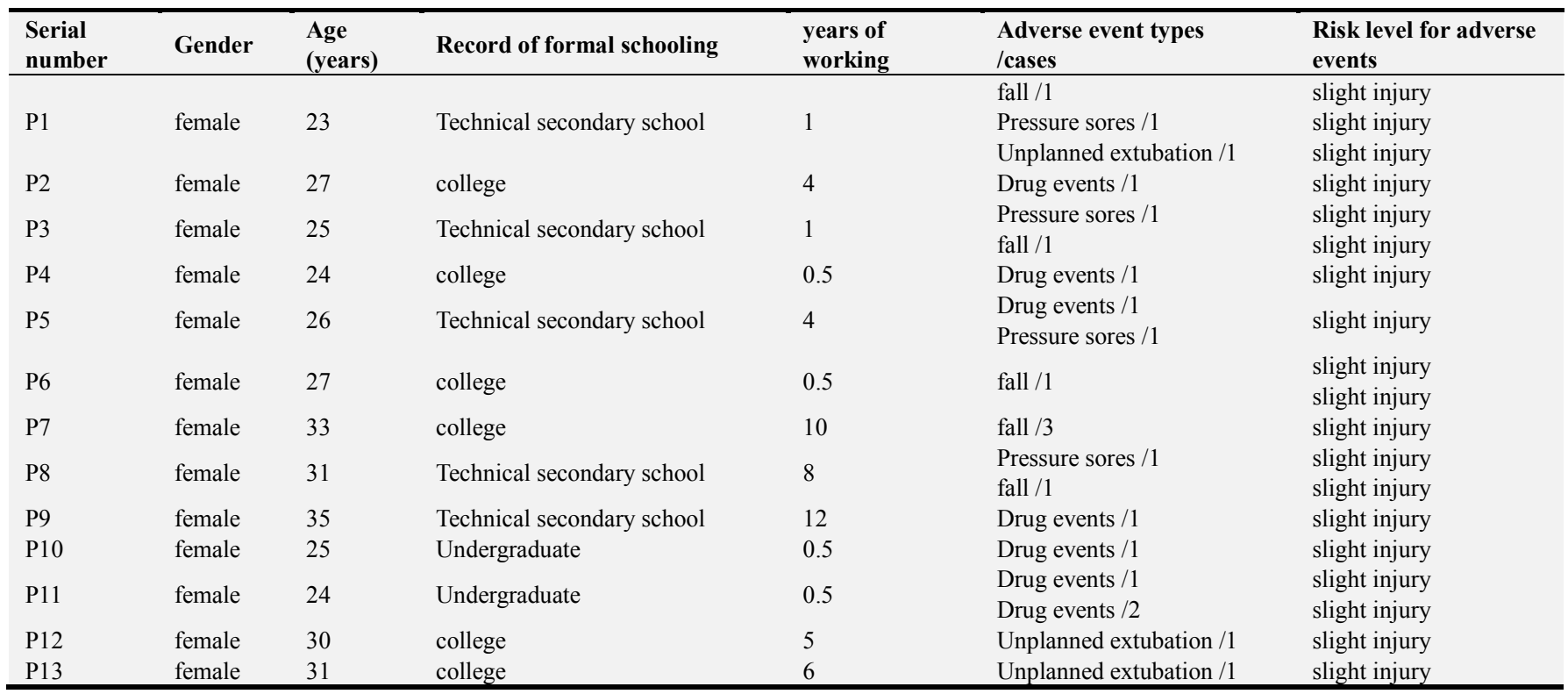

\subsection{The Common Negative Emotions of Nurses After the Occurrence of Adverse Events}

Nurses are also the victims of adverse events. After the occurrence of adverse events, they often show negative psychological emotions such as fear, depression, regret, shame, remorse and depression. Especially when major adverse events occur, the psychological feelings of depression are more intense. P2: "Obviously had done two times fixed, but still pull out by oneself, I am very vexed, want to blame the patient only, but think about, the patient also is not willing, pull out still have to insert back", P3: "It was my fault that I didn't check it carefully. I thought the patient's limbs were ready to move, so I neglected to check the patient's skin.", P3: "It happened three hours after I took over. I have to admit it", P4:"When things happen, I am also very at a loss, can only say that I am responsible for the work", P7: "Until he fell down and broke his femoral head, I really don't want to say anything, I'm in a bad mood just want to get off work". Under the influence of clinical experience and other factors, nurses with low seniority are more likely to be afraid, overwhelmed and even have doubts about their career when something goes wrong. The new entry P11: "I feel very guilty for the physical injury caused by my carelessness to patients, and I wonder whether I am suitable for this position."

\subsection{The Nurse Thinks That the Gibbs Reflective Cycle Is Helpful for Her Growth, Especially for the Staff with Low Seniority}

Most clinical nurses think that the various steps of the "Gibbs Reflective Cycle" are clear in thinking and analysis, Guidelines are provided for adverse events, especially for junior nurses. P10: "At the beginning, I felt very upset when I wrote so many words, but after calm reflection, I think there are still many details that can be improved in the work". P2: "It was a little bit of a stretch at first, but Corey had a rule, so go ahead and write it down. I still think it's a good idea, and it helps us see things clearly." P3: "Exercise and improve my clinical nursing thinking." P5: "At first, I thought that the transfusion was not timely because the shift nurse did not explain clearly. I sat down to comb through the whole process and found that I had also made mistakes". P9: "I think it's good that you asked us to write this. It's good for self-reflection, especially for their junior nurses". It is difficult to analyse cooperative and participatory events such as patient and family factors. The daily life care of domestic patients is mostly participated by relatives, some patients need 24-hour care from relatives, and some nursing work can only be completed through the coordination of hospitals, relatives, patients and nursing staff. In adverse events involving uncontrollable participation factors such as patients or relatives, there will be complaints and unclear understanding of fundamental problems in the process of reflection. The nurses concerned believed that they had completed the relevant work as required, and it was not their responsibility if an adverse event occurred. After the occurrence of an adverse event, they did not reflect on the underlying causes, which was particularly prominent in the adverse event of a fall. P7:"I think is the patient's reason causes, but you can't write the patient, otherwise think my attitude is not right." P6: "I kept looking for my own problems, I was hard to analyse the specific reasons, so I scribbled a few." P1: "I was the only one working on the graveyard shift and couldn't be around patients, If relatives don't cooperate, there's no way." P3: "When taking over the shift, I had explained to the patient clearly, but the patient still did not listen to the instructions, so he decided to get out of bed, which led to his fall." P4: "Upon admission, the patient's relatives and patients had been given health publicity and education to prevent falls, but they still fell." 


\subsection{Nurses Are Afraid of Having a Bad Record for the Rest of Their Careers}

At present, the reporting system of adverse events in all countries adopts the principle of non-punishment, encouraging active reporting to avoid concealing the occurrence of events. However, in this non-punishment culture, nurses still worry about having bad professional records [8]. Especially for the young nurses, the psychological burden is heavier. New nurse P11: "After the occurrence of three adverse drug events, I was particularly worried that the department would dismiss me, and even more worried that reflection would become my record". Eyes will dodge, tone has a pause. New nurse P10: "Adverse events were reported at that time, and there were some psychological concerns if I was required to write a reflection journal".

\section{Discussion}

\subsection{Gibbs Reflective Cycle Can Help Nurses Build Clinical Nursing Thinking}

Since the concept of critical thinking was introduced into the field of nursing in the 1980s, it has been highly valued and regarded as one of the standards to measure the quality of nursing education in many countries [9]. Good critical thinking ability is the basis to ensure that nurses can make rational and effective decisions in future clinical practice [10] Gibbs' reflective cycle is a useful tool to promote reflection: it provides a framework to encourage the narrator to analyse thoughts and feelings, consider alternative solutions, and draw up action plans [11]. The writing of narratives using Gibbs' reflective cycle is an unconventional, innovative method to teach and assess competencies in the affective domain [12]. Studies confirmed that writing profoundly improves levels of empathy [13], their professionalism [14, 15], their respect for the diversity of patients, and their communication skills [15]. Deep understanding of Breaking bad news (BBN) encounters [16]. Demonstrate a significant increase in the nurses' ability to think critically as well as components of engagement, cognitive maturity and innovativeness after implementing CRD based on Graham Gibbs Cycle [6].

This study applies "Gibbs Reflective Cycle" to the reflection of adverse events, most interviewees believe that the theoretical framework is clear and the steps are clear, which is conducive to the analysis and establishment of clinical thinking, especially for nurses with low seniority. Senior nursing work because of rich work experience, the analysis of things more thorough, the effect is not significant.

\subsection{Managers Should Pay Attention to the Negative Psychological Emotions of Clinical Nurses, Care About Nurses and Eliminate Their Concerns.}

When nursing adverse events occur, nurses often experience anxiety, guilt, shame and other adverse experiences, doubt their choice of career, worry about the occurrence of complaints, and some nursing staff will be unable to qualified for the previous post in a short period of time. China's nursing managers focus on the exploration and reason analysis of the reporting system, ignoring the inner guidance of nurses, so the nursing managers should be more concerned about the physical and mental feelings of nurses. At the same time, the purpose of "Gibbs Reflective Cycle" is emphasized in the training to eliminate concerns.

\subsection{Enlightenment to Nursing Work}

The nursing manager should strengthen the training of the knowledge of the circle of reflection, so that the nurse can understand clearly the connotation of each step. Meanwhile, the reflection diary written by the nurse should be modified and supervised, so as to strengthen the understanding of the connotation in practice, so as to avoid the aversion of the nurse [17].

\section{Conclusions}

In this study, qualitative method was used to describe in detail the psychological experience of clinical nurses who used the Gibbs Reflective Cycle for the reflection process of adverse events, Junior nursing staff believe that Gibbs Reflective Cycle is conducive to helping nurses establish clinical nursing thinking and deepen their deep understanding of the causes of adverse events. At the same time, nursing managers should pay attention to the negative psychological reactions of nurses, care for the care of nursing staff, to eliminate their concerns. The shortcomings of this study are that adverse events are mostly caused by nurses with low seniority, so the proportion of the interviewees with high seniority is relatively small, so the seniority of the interviewees is not balanced.

\section{Acknowledgements}

The authors declare no conflict of interest.

\section{References}

[1] Butler, M P, Cassidy I, Quillinan B, etal. Competency assessment methods-toolsand process: a survey of nurse preceptors in Ireland. Nurse Education in Practice, 2011, 11 (5), 298-303.

[2] Tawanwongsri W, Phenwan T Reflective and feedback performances on Thai medical students' patient history-taking skills. BMC Med Educ. 2019 May 14; 19 (1): 141.

[3] Gibbs, G. (1988). Learning by doing: A guide to teaching learning methods. Oxford: Oxford Brookes University.

[4] Song Yanli, Liu Xiaonan, Liu Yang, Gao Min, Zhan Yang, Zhou Yiyi. Application of reflection method in nursing profession [J]. Journal of Jilin Medical College, 2019, 40 (03): 192-193. 
[5] Yang Guoyong, Gao Chunyan, Guo Yan, Han Xu. Research on the application of Gibbs reflection cycle in the teaching of nursing students in operating room [J]. Journal of Nursing Management, 2018, 18 (03): 226-228.

[6] Ardian P, Hariyati RTS, Afifah E. Correlation between implementation case reflection discussion based on the Graham Gibbs Cycle and nurses' critical thinking skills. Enferm Clin. 2019, 29 (2): 588-593

[7] Smith JA, Osborn M. Interpretative phenomenological analysis. In: Smith JA, editor. Qualitative psychology: a practical guide to research methods. London: Sage Publications Ltd; 2003.

[8] Lu Yongli, Wang Peihong, Li Shasha, Li Xia. A qualitative study on the attitude of nursing managers to the second victim in adverse nursing events [J]. Chinese Journal of Nursing, 2019, 54 (08): 1210-1214.

[9] Stewart S, Dempsey LF. A longitudinal study of baccalaureate nursing students'critical thinking dispositions. J Nurs Educ, 2005, 44 (2): 81-84.

[10] Fu Hongqiong, Zhu Dan. Status and analysis of critical thinking teaching mode in higher nursing education in China [J]. Journal of Nursing of Chinese People's Liberation Army, 2005, 22 (2): 58-59.

[11] Oxford Brookes University. Reflective writing: about Gibbs Reflective Cycle, 2016 [cited 2016 Sep 23]. Available from: https://www.brookes.

ac.uk/students/upgrade/study-skills/reflective-writing-gibbs/

[12] Dhaliwal U, Singh S, Singh N. Reflective student narratives: honing professionalism and empathy. Indian J Med Ethics. 2018 Jan-Mar; 3 (1): 9-15.

[13] Chen I, Forbes C. Reflective writing and its impact on empathy in medical education: systematic review. J Educ Eval Health Prof. 2014; 11: 20.

[14] Arntfield SL, Slesar K, Dickson J, Charon R. Narrative medicine as a means of training medical students toward residency competencies. Patient Educ Couns. 2013; 91 (3): 280-6.

[15] Bernard AW, Malone M, Kman NE, Caterino JM, Khandelwal S. Medical student professionalism narratives: a thematic analysisand interdisciplinary comparative investigation. BMC Emerg Med. 2011; 11 (1): 11.

[16] See, reflect, learn more: qualitative analysis of breaking bad news reflective narratives. Karnieli-Miller O, Palombo M, Meitar D. Med Educ. 2018, 52 (5): 497-512.

[17] Fiona Timmins, Maryanne Murphy, Rachel Howe, etal. "I hate Gibb's reflective cycle 1998"(Facebook@2009): Registered nurses experiences of supporting nursing students'reflective practice in the context of student's public commentary Procedia- Social and Behavioral Sciences. 93 (2013): 1371-1375. 